\title{
Near-infrared Polarimetry and Interstellar Magnetic Fields in the Galactic Center
}

\author{
S. Nishiyama ${ }^{1}$, H. Hatano ${ }^{2}$, T. Nagata ${ }^{3}$ and M. Tamura ${ }^{1}$ \\ ${ }^{1}$ National Astronomical Observatory of Japan, Mitaka, Tokyo 181-8588, Japan \\ ${ }^{2}$ Department of Astrophysics, Nagoya University, Nagoya 464-8602, Japan \\ ${ }^{3}$ Department of Astronomy, Kyoto University, Kyoto 606-8502, Japan \\ email: shogo.nishiyama@nao.ac.jp
}

\begin{abstract}
We present a large-scale view of the magnetic field (MF) in the central $3^{\circ} \times 2^{\circ}$ region of our Galaxy. There is a smooth transition of the large-scale MF configuration in this region.
\end{abstract}

Keywords. infrared: ISM, techniques: polarimetric, magnetic fields

We have carried out polarimetric observations using the near-infrared polarimetric camera SIRPOL on the $1.4 \mathrm{~m}$ telescope IRSF, and have obtained a large-scale view of the magnetic field in the central $3^{\circ} \times 2^{\circ}$ region of our Galaxy. We find that near the Galactic plane, the magnetic field is almost parallel to the Galactic plane (i.e., toroidal configuration) but at high Galactic latitudes $\left(|b|>0.4^{\circ}\right)$, the magnetic field is nearly perpendicular to the plane (i.e., poloidal configuration). For more detail, see Nishiyama et al. (2009), Nishiyama et al. (2010).

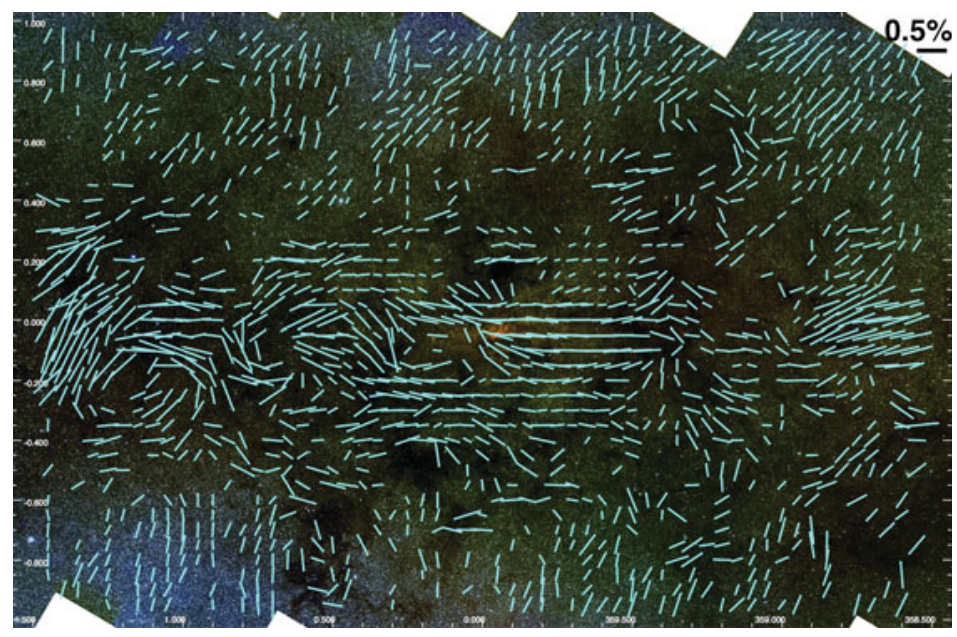

Figure 1. Near-infrared $\left(J, H, K_{S}\right)$ mosaic image of the Galactic center region covering $3^{\circ} \times 2^{\circ}$ in the Galactic coordinate. Observed directions of the magnetic fields at the Galactic center are also plotted with cyan bars whose length indicates the degree of polarization in the $K_{S}$ band.

\section{References}

Nishiyama, S., et al. 2009, ApJ, 690, 1648

Nishiyama, S., et al. 2010, ApJ, 722, L23 